\title{
Toll-like receptor 9-mediated inhibition of apoptosis occurs through suppression of FoxO3a activity and induction of FLIP expression
}

\author{
Eun-Jung Lim ${ }^{1}$, Dae-Weon Park ${ }^{1}$, Jin-Gu Lee ${ }^{1}$, \\ Chu-Hee Lee ${ }^{1}$, Yoe-Sik Bae', \\ Young-Chul Hwang ${ }^{3}$, Jae-Weon Jeong ${ }^{3}$, \\ Byung-Rho $\mathrm{Chin}^{3}$ and Suk-Hwan Baek ${ }^{1,4}$ \\ ${ }^{1}$ Aging-Associated Vascular Disease Research Center \\ Department of Biochemistry and Molecular Biology \\ College of Medicine, Yeungnam University \\ Daegu 705-802, Korea \\ ${ }^{2}$ Department of Biological Science \\ Sungkyunkwan University \\ Suwon 440-746, Korea \\ ${ }^{3}$ Department of Dentistry \\ College of Medicine, Yeungnam University \\ Daegu 705-717, Korea \\ ${ }^{4}$ Corresponding author: Tel, 82-53-620-4523; \\ Fax, 82-53-623-8032; E-mail, sbaek@ med.yu.ac.kr \\ DOI 10.3858/emm.2010.42.10.070
}

Accepted 25 August 2010

Available Online 26 August 2010

Abbreviations: FLIP, FLICE-like inhibitory protein; FoxO, forkhead box O; ODN, oligodeoxynucleotides; PI3K, phosphatidylinositol 3 kinase; TLR9, Toll-like receptor 9

\begin{abstract}
Synthetic oligodeoxynucleotides (ODN) with a CpGmotif are recognized by Toll-like receptor 9 (TLR9) and pleiotropic immune responses are elicited. Stimulation of macrophages with TLR9 agonist prevented apoptosis induced by serum deprivation through increased expression of FLICE-like inhibitory protein (FLIP). CpG ODN-mediated anti-apoptosis depended on the TLR9-Akt-FoxO3a signaling pathway. Inhibition of TLR9 by small interfering (si) RNA or an inhibitor suppressed CpG ODN-mediated anti-apoptosis. Analysis of signaling pathways revealed that the anti-apoptotic effect of CpG ODN required phosphorylation of FoxO3a and its translocation from the nucleus to the cytosol. Overexpression of FoxO3a increased apoptosis induced by serum deprivation and CpG ODN blocked these effects through FLIP expression. In contrast, siRNA knock-down of FoxO3a decreased apop-
\end{abstract}

tosis by serum deprivation. In addition, Akt activation was involved in CpG ODN-induced phosphorylation of FoxO3a, expression of FLIP, and anti-apoptosis. Taken together, these results demonstrate the involvement of Akt-FoxO3a in TLR9-mediated anti-apoptosis and indicate that FoxO3a is a distinct regulator for FLIP expression.

Keywords: Apoptosis; CpG ODN; FLIP; FoxO3a; Toll-like receptor 9

\section{Introduction}

Toll-like receptors (TLRs) recognize a set of conserved molecular structures called pathogenassociated molecular patterns, which allow them to sense innate and adaptive immune responses. Among them, TLR9 is essential for recognition of microbial CpG DNA or synthetic CpG oligonucleotide analogs containing a CPG oligodeoxynucleotide (ODN). CpG DNA activates macrophages, monocytes, and dendritic cells to secrete proinflammatory cytokines (Krieg, 2006; Lim et al., 2007). TLR9 is confined primarily to cells of the immune system and TLR9 is initially localized in the endoplasmic reticulum (Latz et al., 2004). The binding of CpG DNA to TLR9 and the subsequent endosomal maturation are thought to be essential for $\mathrm{CpG}$ DNA-driven immunostimulatory activity (Takeshita et al., 2004). After CpG DNA binding, TLR9 signaling is initiated by recruitment of the adaptor molecule MyD88 followed by the engagement of interleukin (IL)-1R-associated kinases and tumor necrosis factor (TNF)- $\alpha$ receptor (TNFR)-associated factor 6 (Kawai et al., 2004). These complexes can activate the IאB kinase complex and subsequently activate $\mathrm{NF} \kappa \mathrm{B}$-dependent pro-inflammatory cytokines such as TNF- $\alpha$ and IL-1 $\beta$ (Bagchi et al., 2007). TLRs are members of the IL-1R superfamily and share a common activation pathway by their Toll/IL-1R signaling domain (Boraschi and Tagliabue, 2006). Despite this common pathway, TLRs show differences in their rate, intensity, or efficiency of activation by as yet unidentified mechanisms. Selective pathways are triggered by some TLRs; in particular, TLR2, TLR4, and TLR9 can activate 
the phosphatidylinositol 3 kinase (PI3K) pathway (Francois et al., 2005).

Members of the FoxO subfamily of forkhead transcription factors include the mammalian ortholog DAF-16, which regulates longevity in the nematode Caenorhabditis elegans (Lee et al., 2003). Mice and humans possess three highly related FoxO homologs (FoxO1, FoxO3, and FoxO4) with overlapping patterns of expression and transcriptional activities (Biggs et al., 2001). Suppression of FoxO transcriptional activity by Akt-mediated phosphorylation leads to enhanced cell survival (Srinivasan et al., 2005). In conditions in which the Akt survival and growth pathway are activated, FoxO3a is phosphorylated by Akt and exported to the cytoplasm where it is sequestered by the 14-3-3 protein (Caporali et al., 2008). In contrast, unphosphorylated $\mathrm{FoxO} 3 \mathrm{a}$ proteins are active forms and are located in the nucleus where they bind to the promoters of their target genes. Overexpression of a constitutively activated form of FoxO3a leads to apoptosis of many cell types (Cui et al., 2008). The mitochondrial pathway can promote apoptosis in hematopoietic cells after activation of FoxO3a signaling (Dijkers et al., 2002). Additionally, FoxO3a mediates apoptosis by activating pro-apoptotic genes such as Fas ligand, Bim, or TNF-related apoptosis-inducing ligand (TRAIL) (Lam et al., 2006). Even though FoxO3a has generally been considered an inducer of apoptosis, there is no evidence of a relationship between FoxO3a and TLR activation.

The present study investigated the role of FoxO3a transcription factor in TLR9-mediated anti-apoptosis of macrophages. CpG ODN treatment blocked serum deprivation-mediated apoptosis and up-regulated FLIP gene expression. The mechanisms of CpG ODN-induced expression of FLIP via the TLR9-FoxO3a signaling pathway were investigated.

\section{Results}

\section{CpG ODN suppresses apoptosis of macrophages induced by serum starvation}

Since TLR9 is expressed and functional on activated macrophages, it was necessary to confirm that TLR9 engagement on macrophages resulted in altered cell death. Accordingly, apoptosis was examined in TLR9 stimulated and non-stimulated Raw264.7 cells in response to serum deprivation by evaluating the percentage of propidium iodide (PI) or annexin- $\mathrm{V}$ stained cells. After CpG ODN 1668 treatment, a significant and time-dependent decrease in sub $\mathrm{G} 1$ cells was
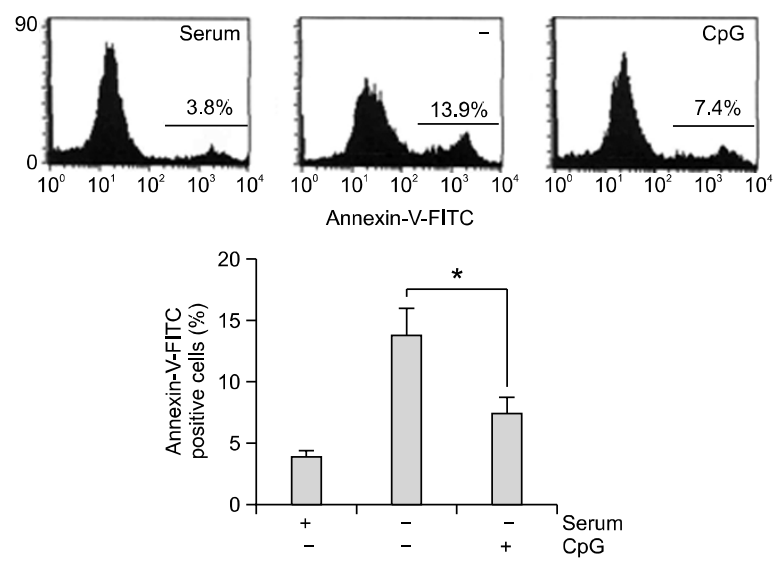

Figure 1. CpG ODN inhibits apoptosis of macrophages induced by serum deprivation. Raw264.7 cells were cultured, changed into serum-free medium, and incubated with serum, medium (-), or $3 \mu \mathrm{M} \mathrm{CpG} \mathrm{ODN} \mathrm{(CpG).}$ Cells were stained with annexin-V FITC and analyzed for the induction of apoptosis by flow cytometry. Each data point represents the mean \pm S.D. of five independent experiments $\left({ }^{*}, P<0.05\right)$.

evident as compared to non-stimulated controls or control ODN 1720 (data not shown). In annexin-V stained cells, a decrease of apoptotic cells in response to CpG ODN was evident (Figure 1). These findings were consistent with the suggestion that TLR9 engagement reduces apoptosis in response to serum deprivation.

\section{CpG ODN regulates apoptosis via TLR9}

Two lines of evidence presently suggested that TLR9 is the receptor that mediates the CpG ODN signal to apoptosis. Firstly, two pharmacological inhibitors of TLR9, CQ or bafilomycin A1 (Bafilo), were used to examine the role that the receptor plays during response in macrophages. Specifically, these drugs were used to measure the effect of TLR9 inhibition on serum deprivationinduced apoptosis. As previously observed, serum deprivation increased apoptosis, and these responses were suppressed by the addition of $\mathrm{CpG}$ ODN. This altered anti-apoptosis response was abrogated when TLR9 inhibitors were added to macrophages (Figure 2A). The second line of evidence resulted from the transfection of cells with a vector expressing TLR9 siRNA or nonspecific control siRNA. RT-PCR indicated the knock-down of TLR9 expression in Raw264.7 cells by TLR9 siRNA (Figure 2B). The effect of TLR9 siRNA on CpG ODN-mediated anti-apoptosis was examined using annexin-V stained cells. TLR9 siRNA recovered the annexin- $V$ staining that had been decreased by CpG ODN (Figure 2C). These results supported the suggestion that TLR9 controls CpG ODN-mediated anti-apoptosis of 

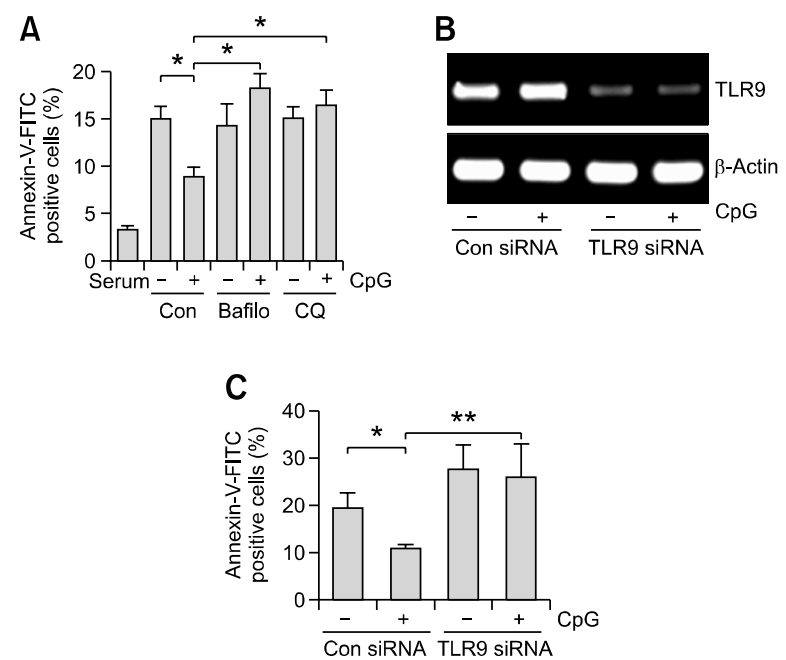

Figure 2. TLR9 mediates anti-apoptosis by CpG ODN. (A) Cells were changed into serum free medium, and pretreated with $5 \mathrm{nM}$ bafilomycin A1 or $5 \mu \mathrm{g} / \mathrm{ml} \mathrm{CQ}$ prior to $\mathrm{CpG}$ ODN stimulation for $12 \mathrm{~h}$. The cells were stained with annexin-V FITC, and analyzed for the induction of apoptosis by flow cytometry. (B, C) Cells were transfected with TLR9 siRNA or control siRNA for $24 \mathrm{~h}$, changed into serum free medium, and incubated with $3 \mu \mathrm{M}$ CpG ODN for $12 \mathrm{~h}$. TLR9 mRNA levels were analyzed by RT-PCR using specific primers for TLR9 or $\beta$-actin (B). The cells were stained with annexin-V FITC (C), and analyzed for the induction of apoptosis by flow cytometry. Each data point represents the mean \pm S.D. of five independent experiments $\left({ }^{*}, P<0.05\right.$, and $\left.{ }^{* *}, P<0.01\right)$.

macrophages.

\section{CpG ODN up-regulates FLIP expression}

The working hypothesis was that TLR9 engagement on macrophages results in the changed expression of proteins with anti-apoptotic properties, and that this represses starvation-induced apoptosis. To explore this, the expression of the anti-apoptotic protein FLIP was ascertained in CpG ODN-treated and non-treated cells. Cultured macrophages were serum deprived for $9 \mathrm{~h}$ prior to transfer to fresh unadulterated medium (control) or medium containing $3 \mu \mathrm{M} \mathrm{CpG} \mathrm{ODN.} \mathrm{Higher} \mathrm{levels}$ of FLIP were observed in cells exposed to CpG ODN (Figure 3A). Next, the change of FLIP expression by TLR9 inhibitors was assessed. Two kinds of TLR9 inhibitors blocked CpG ODNinduced FLIP expression (Figure 3B). The antiapoptosis effects of CpG ODN and the expression change of FLIP in the cell line with peritoneal primary macrophages separated from mice were confirmed. CpG ODN inhibited serum deprivationinduced apoptosis in peritoneal macrophages. In addition, $\mathrm{CpG}$ ODN also increased the expression of FLIP (Figures 4A and 4B).
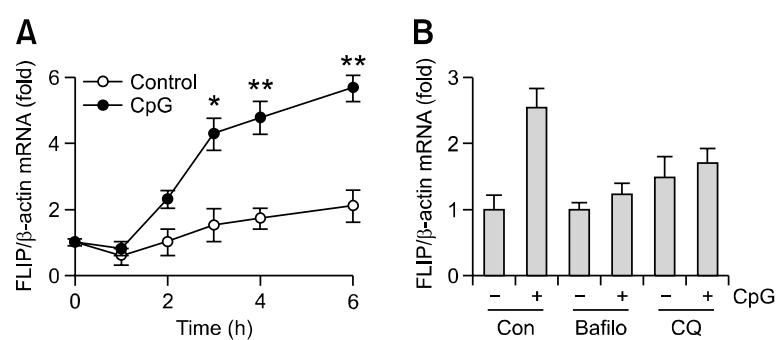

Figure 3. FLIP is involved in CpG ODN-mediated anti-apoptosis. (A) Macrophages were changed into serum-free medium and stimulated with $3 \mu \mathrm{M}$ CpG ODN for the indicated times. Total RNA was then isolated from the cells and subjected to real-time RT-PCR. The graph represents the fold changes of FLIP mRNA after cells were treated with $3 \mu \mathrm{M} \mathrm{CpG}$ ODN. (B) Macrophages were starved for $9 \mathrm{~h}$ and pretreated with $5 \mathrm{nM}$ bafilomycin $\mathrm{A} 1$ or $5 \mu \mathrm{g} / \mathrm{ml} \mathrm{CQ}$ prior to $\mathrm{CpG}$ ODN stimulation for $6 \mathrm{~h}$. Total RNA was then isolated from the cells and subjected to real-time RT-PCR for FLIP mRNA expression. Each data point represents the mean \pm S.D. of four independent experiments $\left({ }^{*}, P<0.05\right.$, and $\left.{ }^{* *}, P<0.01\right)$.

\section{CpG ODN induces phosphorylation of FoxO3a via TLR9}

To assess the downstream intracellular signaling pathways that mediate the anti-apoptosis of TLR9, the activation of FoxO3a, which regulates cell survival was studied, and Western blot analysis was employed to study FoxO3a phosphorylation. As shown in Figure 5A, incubation of macrophages with CpG ODN resulted in phosphorylation of FoxO3a at threonine $32\left(\mathrm{~T}^{32}\right)$. Phosphorylation was rapid, reaching a peak within 40 min. FoxO3a phosphorylation at serine $253\left(\mathrm{~S}^{253}\right)$ was also increased by CpG ODN treatment. Western blot analysis demonstrated $\mathrm{FoxO} 3 \mathrm{a}$ translocation from the nucleus to the cytosol upon $\mathrm{CpG}$ ODN stimulation (Figure 5B). In addition, CpG ODNmediated phosphorylation of FoxO3a was blocked by pretreatment with the TLR9 inhibitors or by TLR9 siRNA (Figures $5 \mathrm{C}$ and $5 \mathrm{D}$ ). As a positive
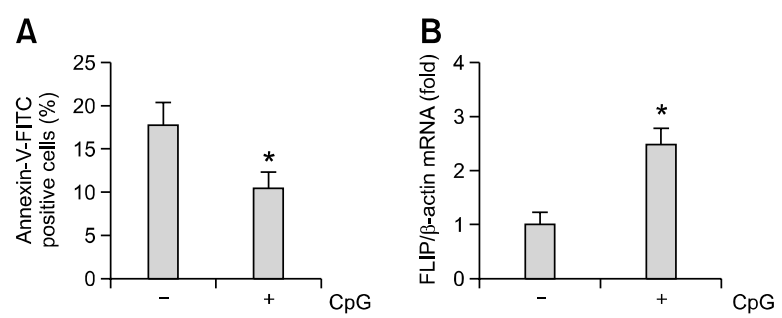

Figure 4. CpG ODN inhibits apoptosis, and regulates FLIP expressions of mice peritoneal macrophages. (A) Mice peritoneal macrophages were isolated, changed into serum-free medium, and incubated with $3 \mu \mathrm{M}$ CpG ODN. Cells were stained with annexin-V and analyzed for the production of apoptosis by flow cytometry. (B) Primary macrophages were changed into serum free medium and stimulated with $3 \mu \mathrm{M}$ CpG ODN. Total RNA was then isolated from the cells and subjected to real-time RT-PCR for FLIP expression. Each data point represents the mean \pm S.D. of four independent experiments $\left({ }^{*}, P<0.05\right)$. 
A

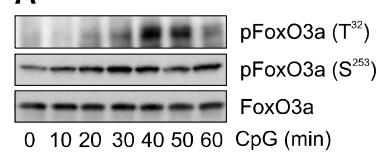

B

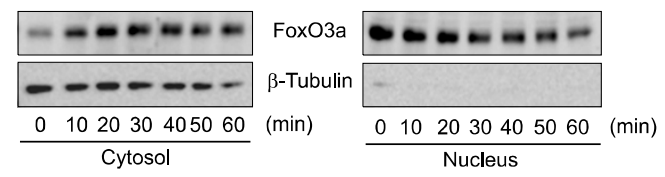

C

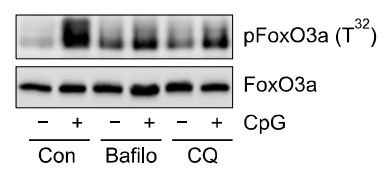

D

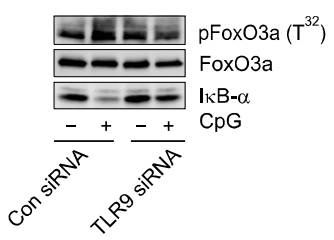

Figure 5. $\mathrm{CpG} O D N$ regulates phosphorylation and localization of FoxO3a through TLR9 activation. (A, B) Macrophages were starved serum for $12 \mathrm{~h}$ and treated with $3 \mu \mathrm{M} \mathrm{CpG}$ ODN for the indicated times. FoxO3a phosphorylation was then assessed by Western blotting using anti-FoxO3a or anti-phospho FoxO3a $\left(\mathrm{T}^{32}\right.$ or $\left.\mathrm{S}^{253}\right) \mathrm{Ab}$. Representative blots are shown (A). Part of cells were separated into the cytosol and nuclear fractions, and analyzed for FoxO3a expression using anti-FoxO3a $\mathrm{Ab}$ or $\beta$-tubulin (B). (C) Cells were pretreated with TLR inhibitors $(5 \mathrm{nM}$ bafilomycin $\mathrm{A} 1,5 \mu \mathrm{g} / \mathrm{ml} \mathrm{CQ}$ ) for $1 \mathrm{~h}$ and stimulated with $\mathrm{CpG}$ ODN for 30 min. FoxO3a phosphorylation was then analyzed by Western blotting using anti-FoxO3a and anti-phospho FoxO3a Ab. (D) Cells were transfected with TLR9 siRNA or control siRNA for $24 \mathrm{~h}$. Cells were incubated in free medium for $12 \mathrm{~h}$, and stimulated with $3 \mu \mathrm{M} \mathrm{CpG} \mathrm{ODN} \mathrm{for} 30 \mathrm{~min}$. FoxO3a phosphorylation was then analyzed by Western blotting using anti-FoxO3a and anti-phospho FoxO3a Ab.

control, CpG ODN-induced $I_{\kappa} \mathrm{B}-\alpha$ degradation (which results in the activation of $N F \kappa B$ ) was present and could be blocked by TLR9 siRNA. These data indicate that CpG ODN induces phosphorylation of $\mathrm{FoxO} 3 \mathrm{a}$ in macrophages through the TLR9-dependent signaling pathway.

\section{CpG ODN regulates FLIP by the FoxO3a-dependent pathway}

Having demonstrated that $\mathrm{CpG}$ ODN regulates phosphorylation of $\mathrm{FoxO} 3 \mathrm{a}$ and the expressions of FLIP, the interaction of FoxO3a with FLIP was assessed in FoxO3a overexpressing macrophages. To prove that $\mathrm{FoxO} 3 \mathrm{a}$ principally regulates the transcription of FLIP, cells were transduced with lentivirus expressing vector or FoxO3a, and FLIP mRNA expression was assessed by real-time $\mathrm{PCR}$. Overexpression of FoxO3a protein increased in lentivirus-mediated FoxO3a transduced cells compared to vector transduced cells (Figure 6A). Overexpression of FoxO3a slightly decreased the FLIP mRNA level, while CpG ODN treatment blocked the stimulatory effect on FLIP expression
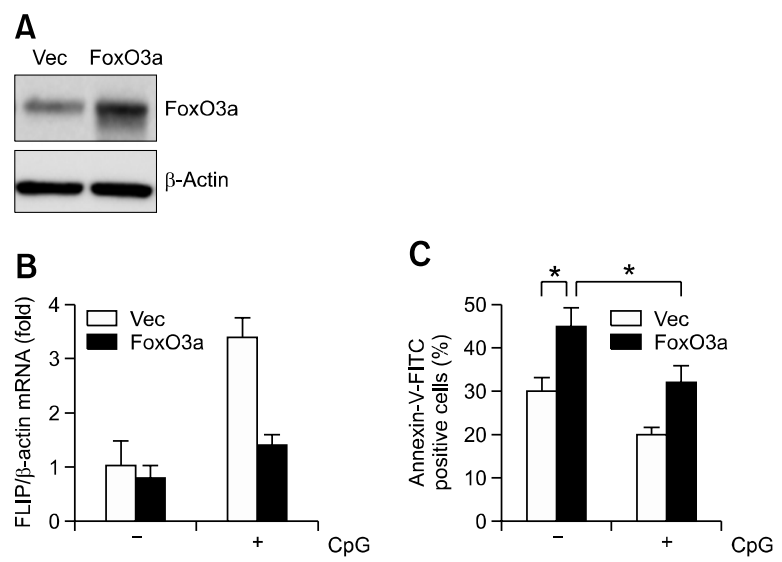

Figure 6. FoxO3a overexpression increases serum-deprived apoptosis. Cells were stably transfected with a control vector (pLenti/V5-D-TOPO) or cDNA encoding FoxO3a. (A) FoxO3a expression was assessed by Western blotting using anti-FoxO3a Ab. (B) Control vector or FoxO3a overexpressing cells were changed into serum-free medium. After $9 \mathrm{~h}$, cells were incubated with $3 \mu \mathrm{M} \mathrm{CpG}$ ODN for $6 \mathrm{~h}$, and FLIP mRNA levels were assessed by real-time RT-PCR. (C) Vector or FoxO3a overexpressing cells were changed with serum-free medium and treated with $3 \mu \mathrm{M}$ CpG ODN for $12 \mathrm{~h}$, cells were stained with annexin-V FITC, and analyzed for the induction of apoptosis by flow cytometry. Each data point represents the mean \pm S.D. of four independent experiments $\left({ }^{*}, P<0.05\right)$.

(Figure 6B). These results suggested that $\mathrm{CpG}$ ODN could up-regulate FLIP expression via the FoxO3a-dependent signaling pathway. FACS analysis for annexin- $V$ positive cells showed that overexpression of FoxO3a increased apoptosis by more than $16 \%$ compared with vector control cells. However, incubation with CpG ODN reduced apoptotic cells in both the control vector or FoxO3 overexpressing cells (Figure $6 \mathrm{C}$ ). The results obtained by FoxO3a overexpression were confirmed by the use of FoxO3a siRNA. FoxO3a siRNA abrogated the protein expression of FoxO3a (Figure 7A). The expression of FLIP induced by CpG ODN was compared in the both control and FoxO3a siRNA cells. CpG ODN increased the expression of FLIP more in FoxO3a siRNA cells than in the control siRNA cells (Figure 7B). Apoptosis was also significantly inhibited more in the FoxO3a siRNA cells (Figure 7C). Taken together, these results supported the suggestion that FoxO3a controls the expression of FLIP, thereby controlling apoptosis.

\section{Akt is an upstream molecule for FoxO3a-mediated FLIP and apoptosis regulation}

The next experiments assessed the importance of Akt pathway in FLIP expression and anti-apoptosis, which is mediated by CpG ODN. Probing of Akt phosphorylation by CpG ODN produced a peak at $30 \mathrm{~min}$ (Figure 8A). LY294002, a PI3K-Akt 
A
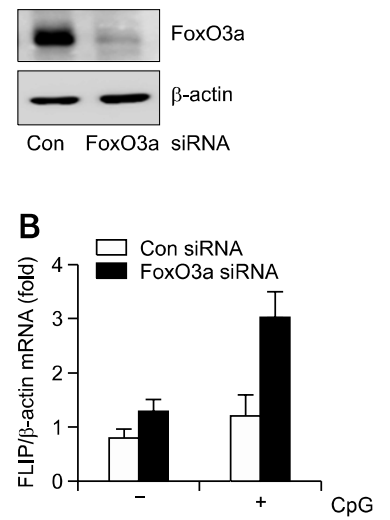

C

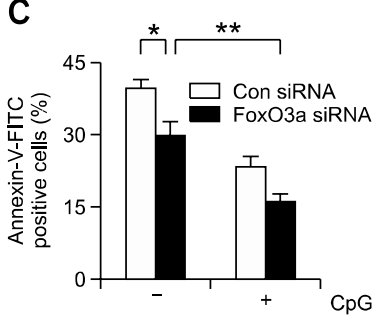

Figure 7. FoxO3a siRNA decreases serum-deprivated apoptosis. Cells were transfected with a control siRNA or FoxO3a siRNA. (A) FoxO3a expression was assessed by Western blotting using anti-FoxO3a $\mathrm{Ab}$. (B) Control or FoxO3a siRNA cells were changed into serum-free medium, After $9 \mathrm{~h}$, cells were incubated with $3 \mu \mathrm{M} \mathrm{CpG}$ ODN for $6 \mathrm{~h}$, and FLIP mRNA levels were assessed by real-time RT-PCR. (C) siRNA-treated cells were changed with serum free media and treated with $3 \mu \mathrm{M} \mathrm{CpG}$ ODN for $12 \mathrm{~h}$, cells were stained with annexin-V FITC, and analyzed for the induction of apoptosis by flow cytometry. Each data point represents the mean \pm S.D. of four independent experiments $\left({ }^{*}, P<0.05\right.$, and ${ }^{* *}$, $P<0.01)$.

pathway inhibitor, inhibited $\mathrm{FoxO} 3 \mathrm{a}$ inactivation by CpG ODN (Figure 8B). Next, apoptosis and FLIP expression in the presence of LY294002 was determined. Treatment of CpG ODN after serum starvation showed time-dependent increase in FLIP expression compared to the non-CpG ODN group. However, pretreatment of LY294002 diminished the increased FLIP expression (Figure $8 \mathrm{C})$. Similar results were shown in apoptosis as well. These results were consistent with increased apoptosis under serum starvation and decreased apoptosis upon CpG ODN treatment. However, LY294002 recovered the diminished apoptosis by CpG ODN (Figure 8D). These results supported the suggestion that Akt is activated by $\mathrm{CpG}$ ODN, which inactivates $\mathrm{FoxO} 3 \mathrm{a}$ and increases FLIP expression, eventually inhibiting apoptosis by serum starvation.

\section{Discussion}

CpG ODN is able to protect B-cells and macrophages against apoptosis (Kuo et al., 2006). With this as a basis, to expand the control mechanism of anti-apoptosis by CpG ODN, the present study has confirmed that TLR9 engagement protects against serum deprivationinduced apoptosis. Furthermore, it is demonstrated for the first time that CpG ODN induces an increase in FoxO3a phosphorylation. This pro-
A
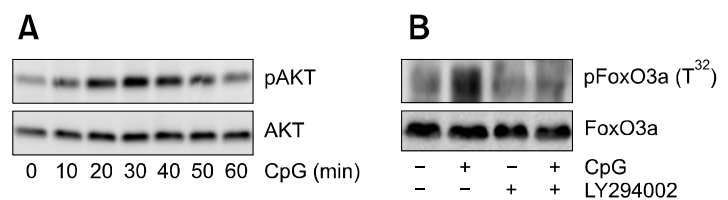

C

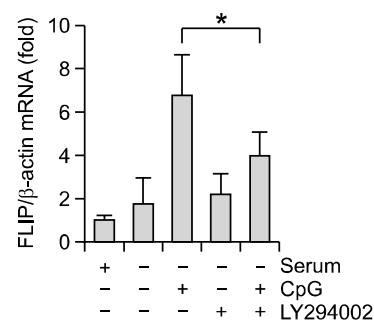

D

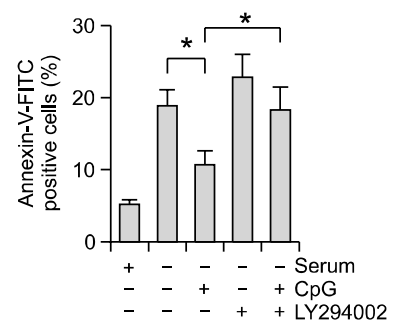

Figure 8. LY294002 is inhibited CpG ODN-mediated anti-apoptosis through FLIP expression. (A) Cells were serum starved for $12 \mathrm{~h}$, incubated with $3 \mu \mathrm{M} \mathrm{CpG}$ ODN for the indicated times, and Akt phosphorylation was assessed by Western blotting using Akt and phospho Akt Ab. (B) Cells were incubated with $3 \mu \mathrm{M} \mathrm{CpG}$ ODN for $30 \mathrm{~min}$ in the absence or presence of $10 \mu \mathrm{M} \mathrm{LY} 294002$. FoxO3a phosphorylation was then assessed by Western blotting using FoxO3a and phospho FoxO3a $\mathrm{Ab}$. (C) Cells were serum starved for $12 \mathrm{~h}$, pretreated with $10 \mu \mathrm{M} \mathrm{LY} 294002$, and incubated with $3 \mu \mathrm{M} \mathrm{CpG}$ ODN. FLIP mRNA levels were assessed by real-time RT-PCR. (D) Cells were changed into serum-free medium, pretreated with $10 \mu \mathrm{M} \mathrm{LY} 294002$, and incubated with $3 \mu \mathrm{M} \mathrm{CpG} \mathrm{ODN}$ for 12 h. Cells were stained with annexin-V FITC, and analyzed for the induction of apoptosis by flow cytometry. Each data point represents the mean \pm S.D. of four independent experiments $\left({ }^{*}, P<0.05\right)$.

tective effect is controlled by increased FLIP expression in $\mathrm{CpG}$ ODN-stimulated macrophages. The anti-apoptotic effects of CpG ODN-stimulation require the participation of FoxO3a. Taken together, these results are consistent with the suggestion that TLR9 triggers the transcription factor FoxO3a and that the regulation of FLIP may contribute to the anti-apoptotic effect.

TLRs including TLR9 play a central role in innate immunity by mediating pathogen recognition (Hong-Geller et al., 2008). CpG ODN is the most well known TLR9 agonist. The study of CpG ODN was mostly focused on innate immunity in various immune cells. However, recent studies show the possibility of applying $\mathrm{CpG}$ ODN on cancer therapy and its other functions as well. For example, CpG ODN was suggested in chemotherapy and radiation therapy as an enhancer of anti-tumor activity (Weigel et al., 2003; Meng et al., 2005). The function of CpG ODN 2006 is one example for enhancing anti-tumor activity by rituximab (Leonard et al., 2007). However, the mechanism to how CpG ODN regulates cancer cells is not clear. We hypothesized that CpG ODN would regulate cancer cells through apoptosis process, and proceeded our experiment. Previous studies have demonstrated a role of TLR9 in mediating the effects of cell survival including macrophages. The 
present study also observed that treatment of macrophages with TLR9 agonists strongly obviates apoptosis. Many groups have investigated the regulatory proteins involved in cell survival, but the specific proteins involved in the response to TLR9 agonist have remained to be clarified. Expression of several apoptosis-related genes is controlled by TLR9 activation. In particular, increased phosphorylation of $\mathrm{Mcl}-1, \mathrm{~A} 1$, and Bad has been implicated in polymorphonuclear leukocyte survival induced by CpG ODN (Francois et al., 2005). In addition, $\mathrm{CpG}$ ODN promotes cell survival via up-regulation of $\mathrm{Hsp} 70$ to increase $\mathrm{Bcl}-\mathrm{xL}$ and decrease apoptosis-inducing factor translocation (Kuo et al., 2006). Furthermore, Hsp90 $\beta$ is also involved in the TLR9 anti-apoptotic effect (Kuo et al., 2007). Appropriately, the present study tested the effect of TLR9 agonist on cellular levels of many proteins participating in apoptotic pathways. Among those possible regulatory factors, the results identify FLIP as an important regulation of macrophages to apoptosis.

Although a number of proteins involved in apoptosis signaling were unaffected, FLIP protein expression was induced in cells treated with $\mathrm{CpG}$ ODN. FLIP inhibits death receptor-induced apoptosis by interacting with the receptor-associated initiator caspase-8 (Wang et al., 2005). FLIP is a protein similar to caspase-8, but which lacks a catalytic domain, and which inhibits the activation mediated by Fas associated protein with death domain (FADD) protein by competitively interacting with caspase-8, (Thome et al., 1997). However, a recent report demonstrated that FLIP activates caspase-8 through heterodimerization (Chang et al., 2002), suggesting that FLIP plays a dual role by activating or inhibiting caspase-8. The present study provides evidence of a definitive role of FLIP in suppressing serum deprivation-induced apoptosis. Up-regulation of FLIP by CpG ODN protected against serum deprivation-induced apoptosis. This protection was not the result of nonspecific inhibition, as specific down-regulation of TLR9 with antisense oligonucleotides or pharmacological inhibition of TLR9 activation blocked the increase of FLIP expression and did not protect from apoptosis. The heightened levels of FLIP expression might be adequate to suppress the apoptosis induced by serum deprivation. The current data is the first to demonstrate that the levels of FLIP directly sensitize macrophages to the anti-apoptotic effects of CpG ODN.

As a transcription factor affecting potentially the expression of many genes, nuclear factor kappa $B$ $(N F \kappa B)$ may favor cell survival by up-regulating gene products with anti-apoptotic properties or down-regulating pro-apoptotic factors (Xu et al., 2008). The up-regulation of FLIP expression following $N F_{\kappa} B$ activation and its involvement in preventing apoptosis has been observed after stimulation by CD40L, lipopolysaccharide, and TNF (Xiao et al., 2002). Furthermore, it has been suggested that the activation of NF $\kappa B$ via the TLR2 signaling pathway and the subsequent induction of FLIP expression are able to protect cells from FasL-induced apoptosis (Loeuillet et al., 2006). Recognition of TLR9 by CpG ODN is also followed by $N_{\kappa} \mathrm{B}$ activation (Takeshita et al., 2004). Despite this, expression of several apoptosis-regulating genes is controlled by other transcription factors including FoxO (Fu and Tindall, 2008).

Previous studies have suggested that Akt activation negatively regulates FoxO transcription factors (Haegebarth et al., 2006), and that the direct phosphorylation of Akt inhibits transcriptional activation of FoxO3a (Burgering and Medema, 2003). In addition, the PI3K/Akt pathway has been reported as a very important signaling molecule in the anti-apoptosis effects of CpG ODN (Kuo et al., 2006). Furthermore, because Akt activity prevent the induction of apoptosis by cytokines, growth factors, and cellular stress (Bao et al., 2005), the effect of Akt pathway on FoxO3a activation, FLIP expression, and apoptosis was assessed. Use of the pharmacological inhibitor LY294002 enabled the examination of the role of the Akt signaling pathway in the regulation of the FoxO3a. The data shows that Akt inhibition results in a significant decrease in the expression of FLIP gene in these cells. Furthermore, direct evidence was obtained by overexpressing or siRNA methods of FoxO3a. The data demonstrate that CpG ODN treatment decreases FLIP expression in FoxO3a overexpressing cells compared to vector control cells. FoxO3a siRNA cells demonstrate opposite results compared to overexpressing cells. Together, these data strongly support the conclusion that FoxO3a is a transcription factor for FLIP regulation in response to $\mathrm{CpG}$ ODN. However, the apoptosis regulatory proteins affected by FoxO3a activity are not limited to FLIP. For example, previous studies have demonstrated a link between FoxO3a and expression of the cell cycle inhibitor/apoptotic gene, p21 and p27 (Hauck et al., 2007). Therefore, the effects of CpG ODN-mediated p21 and p27 expression were presently examined. As expected, CpG ODN significantly inhibited endogenous p21 and p27 protein expression (data not shown).

Currently, the mechanism by which FoxO3a inhibits FLIP expression is unknown. Previously, Skurk et al. (2004) reported that FoxO3a transcription factor inhibits FLIP expression. They 
suggested two possible mechanisms contributing to FLIP down-regulation by FoxO3a: one was through FLIP cleavage by regulating protein level, and the other involved regulation of the mRNA level. The present results are consistent with FoxO3a-mediated inhibition of FLIP mRNA expression. However, the protein level regulation by caspase-dependent FLIP cleavage suggested by Skurk et al. (2004) could not be verified. In other words, CpG ODN did not have effect on the activation increase of caspase-8 and -9 by starvation (data not shown). Therefore, further research on the down-regulation of FLIP by FoxO3a is necessary.

\section{Methods}

\section{Reagent and antibodies}

Cell culture reagents were obtained from Life Technologies (Grand Island, NY). Fetal bovine serum (FBS) was obtained from Thermo Scientific HyClone (Logan, UT). Chloroquine (CQ) was obtained from Sigma-Aldrich (St Louis, MO). Phosphorothioated unmethylated $\mathrm{CpG}$ ODN 1668 (B-class, TCCATGACGTTCCTGATGCT, endotoxinfree) and control ODN 1720 (TCCATGAGCTTCCTGATGCT, inactive control for CpG ODN 1668) were purchased from Genotech (Daejeon, Korea), Annexin-V-fluorescein isothiocyanate (FITC) staining kits were purchased from BD pharmingen (San Diego, CA). RNA reverse transcription-polymerase chain reaction (RT-PCR) core kit was purchased from Axygen Biosciences (Union City, CA). Antibody $(\mathrm{Ab})$ against phospho-FoxO3a $\left(\mathrm{T}^{32}\right.$ or $\left.\mathrm{S}^{253}\right)$ and FoxO3a were purchased from Cell Signaling Technology (Beverly, MA). Bafilomycin A1 (Bafilo) was purchased from Calbiochem (San Diego, CA).

\section{Cell culture}

The Raw264.7 macrophage cell line was obtained from the American Type Culture Collection (Manassas, VA). Cells were grown in Dulbecco's modified Eagle medium (DMEM; Invitrogen, Carlsbad, CA) containing $10 \%$ fetal bovine serum, $2 \mu \mathrm{mol} / \mathrm{L}$ L-glutamine, $10 \mathrm{units} / \mathrm{ml}$ penicillin, and 10 $\mu \mathrm{g} / \mathrm{ml}$ streptomycin at $37^{\circ} \mathrm{C}$ in a humidified atmosphere under $5 \% \mathrm{CO}_{2}$. Cells were treated with synthetic $\mathrm{CpG}$ ODN for various times. Mouse primary peritoneal macrophages were isolated from C57BL/6 mice by lavage using cold-phosphate buffered saline (PBS). Following harvest, cells were spun down and incubated in wells overnight to facilitate cell attachment prior to washing the cells once with fresh full-strength medium.

\section{Fluorescence-activated cell sorting (FACS) analysis}

Apoptosis was quantified by measurement of externalized phosphatidylserine residues as detected using annexin-VFITC. Cells were collected and washed with ice-cold PBS and then suspended in $500 \mu \mathrm{l}$ of annexin- $\mathrm{V}$ binding buffer.
A $100 \mu$ aliquot was taken, $5 \mu$ of annexin-V-FITC was added, and the mixture was incubated for $15 \mathrm{~min}$ at room temperature in the dark. After the addition of $400 \mu \mathrm{l}$ of binding buffer, the cells were processed by the flow cytometer and analyzed using CellQuest software (BD Biosciences, Franklin Lakes, NJ). The results are shown as a histogram with annexin- $V$ positive cells calculated as apoptotic cells.

\section{Western blot analysis}

Cells $\left(1 \times 10^{6}\right)$ were plated in wells and treated with CpG ODN in the presence or absence of inhibitor. They were then washed with ice-cold PBS, trypsinized, and pelleted at $700 \times \mathrm{g}$. Cell pellets were resuspended in lysis buffer comprised of $50 \mathrm{mM}$ Tris- $\mathrm{HCl}(\mathrm{pH} 8.0), 5 \mathrm{mM}$ EDTA, 150 $\mathrm{mM} \mathrm{NaCl}, 0.5 \%$ Nonidet P-40, $1 \mathrm{mM}$ phenylmethanesulfonylfluoride, as well as a protease inhibitor cocktail. The preparations were then cleared by centrifugation and supernatants were saved as cell lysates. Proteins were separated by $8 \%$ reducing sodium dodecyl sulfatepolyacrylamide gel electrophoresis and immunoblotted in $20 \%$ methanol, $25 \mathrm{mM}$ Tris, and $192 \mathrm{mM}$ glycine onto nitrocellulose membranes. Membranes were then blocked with $5 \%$ non-fat dry milk in TTBS $(25 \mathrm{mM}$ Tris- $\mathrm{HCl}, 150$ $\mathrm{mM} \mathrm{NaCl}$, and $0.2 \%$ Tween-20) and incubated with primary $\mathrm{Ab}$ for $4 \mathrm{~h}$. Subsequently, membranes were washed, incubated for $1 \mathrm{~h}$ with secondary $\mathrm{Ab}$ conjugated to horseradish peroxidase, rewashed, and finally developed using an enhanced chemiluminescence system (GE Healthcare, UK).

\section{FoxO3a and Akt phosphorylation assays}

Cells were treated with CpG ODN in the presence or absence of various inhibitors, lysed, and examined by Western blotting as described above. The membranes so obtained were probed with $\mathrm{Ab}$ against phosphorylated FoxO3a or phosphorylated Akt, and blots were stripped and reprobed with $A b$ recognizing both phosphorylated and total proteins, to demonstrate equal loading.

\section{Cytosol and nucleus fractionation}

To determine the subcellular localization of FoxO3a in cells, nuclear fractionation and Western blot analyses were used to detect the amount of FoxO3a in nuclei and cytoplasm, respectively. After CpG ODN treatment, cells were lysed in ice-cold hypotonic buffer containing $10 \mathrm{mM}$ HEPES, pH 7.9, $10 \mathrm{mM} \mathrm{KCl}, 0.1 \mathrm{mM}$ EDTA, $0.1 \mathrm{mM}$ EGTA, $1 \mathrm{mM}$ dithiothreitol, and a cocktail of protease inhibitors. After centrifugation at $12,000 \times \mathrm{g}$ for $30 \mathrm{~s}$, the supernatant was collected as the cytosolic fraction. The pellet was further extracted using buffer containing $20 \mathrm{mM}$ HEPES, $0.4 \mathrm{M} \mathrm{NaCl}, 1 \mathrm{mM}$ EDTA, $1 \mathrm{mM}$ EGTA, $1 \mathrm{mM}$ dithiothreitol, and a cocktail of protease inhibitors at $4^{\circ} \mathrm{C}$. Soluble nuclear proteins were collected after centrifugation at $12,000 \times \mathrm{g}$ for $10 \mathrm{~min}$.

\section{Real-time RT-PCR}

Total RNA was extracted from cells using Trizol reagent 
(Invitrogen). Total RNA $(1 \mu \mathrm{g})$ was used as a template to make first strand cDNA by oligo-dT priming using a reverse transcriptase system (Promega, Madison, WI). Real-time RT-PCR was performed using a LightCycler 1.5 (Roche Diagnostics, Almere, Netherlands) with SYBR-Green I as the florescent dye according to the manufacturer's instructions. The synthetic gene-specific primer sets used for PCR were: (1) FLIP forward primer, 5'-AGTGAGGCGGTTTGACCTTCT-3', and reverse primer, 5'-TTGCCTCTGCCTGTGTAA -3', which amplified 189-bp of mouse FLIP cDNA; (2) TLR9 forward primer, 5'-GGGCCCATTGTGATGAACC3', and reverse primer, 5'-GCTGCCACACTTCACACCAT3', which amplified 141-bp of mouse TLR9 cDNA; (3) $\beta$-actin forward primer, 5'-AGAGGGAAATCGTGCGTGAC3', and reverse primer, 5'-CAATAGTGATGACCTGGCCGT-3', which amplified 137-bp of mouse $\beta$-actin cDNA. Cycling conditions were $94^{\circ} \mathrm{C}$ for $10 \mathrm{~min}$, followed by 45 cycles of $95^{\circ} \mathrm{C}$ for $10 \mathrm{~s}, 62^{\circ} \mathrm{C}$ for $5 \mathrm{~s}$, and $72^{\circ} \mathrm{C}$ for $8 \mathrm{~s}$. For quantification, target genes were normalized versus $\beta$ actin.

\section{Knock-down of TLR9 or FoxO3a using small interfering RNA (siRNA)}

Oligonucleotides corresponding to the mouse TLR9 siRNA sequence 5'-CCAACAUCCUGGUUCUAGAUGCUAA-3' and mouse FoxO3a siRNA sequence 5'-UGAUGAUCCACCAAGAGCUCUUGCC-3' were purchased from Invitrogen. A control siRNA was also purchased and used. For transfection, $2 \times 10^{6}$ Raw264.7 cells were resuspended in a nucleoporator buffer (Lonza Cologne AG, Köln, Germany) with 100-200 pmole siRNA. Cells were nucleoporated according to the manufacturer's protocol and allowed to knock-down the above genes for $24 \mathrm{~h}$.

\section{Overexpression of FoxO3a}

For protein expression in cells, a vector encoding a FoxO3a protein (pLenti6/V5-D-TOPO-FoxO3a) was generously provided by Dr. Kim (Yeungnam University, South Korea). Cells were transfected with control vector, wild type FoxO3a for $24 \mathrm{~h}$ and fresh medium was added. To obtain a stable cell line, cells transfected with the cDNA were cloned by serial dilution in a 96-well plate in a culture medium with selected antibiotics. The sub-culturing was continued for 4 weeks, and then wells representing a single colony were selected and the expression was confirmed using its protein level as determined by Western blot analysis.

\section{Acknowledgements}

This work was supported by the Korea Science and Engineering Foundation (KOSEF) grant funded by the Korea government (MEST) (2010-0001243) and by Midcareer Researcher Program through NRF grant funded by the MEST (2009-0083759).

\section{References}

Bagchi A, Herrup EA, Warren HS, Trigilio J, Shin HS, Valentine C, Hellman J. MyD88-dependent and MyD88independent pathways in synergy, priming, and tolerance between TLR agonists. J Immunol 2007;178:1164-71

Bao S, Wang Y, Sweeney P, Chaudhuri A, Doseff Al, Marsh $\mathrm{CB}$, Knoell DL. Keratinocyte growth factor induces Akt kinase activity and inhibits Fas-mediated apoptosis in A549 lung epithelial cells. Am J Physiol Lung Cell Mol Physiol 2005; 288:L36-42

Biggs WH 3rd, Cavenee WK, Arden KC. Identification and characterization of members of the FKHR (FOX O) subclass of winged-helix transcription factors in the mouse. Mamm Genome 2001;12:416-25

Boraschi D, Tagliabue A. The interleukin-1 receptor family Vitam Horm 2006;74:229-54

Burgering BM, Medema RH. Decisions on life and death: FOXO Forkhead transcription factors are in command when PKB/Akt is off duty. J Leukoc Biol 2003;73:689-701

Caporali A, Sala-Newby GB, Meloni M, Graiani G, Pani E, Cristofaro B, Newby AC, Madeddu P, Emanueli C. Identification of the prosurvival activity of nerve growth factor on cardiac myocytes. Cell Death Differ 2008;15:299-311

Chang DW, Xing Z, Pan Y, Algeciras-Schimnich A, Barnhart $B C$, Yaish-Ohad S, Peter ME, Yang X. c-FLIP $(L)$ is a dual function regulator for caspase-8 activation and CD95mediated apoptosis. EMBO J 2002;21:3704-14

Cui $\mathrm{M}$, Huang $\mathrm{Y}$, Zhao $\mathrm{Y}$, Zheng J. Transcription factor FOXO3a mediates apoptosis in HIV-1-infected macrophages. J Immunol 2008;180:898-906

Dijkers PF, Birkenkamp KU, Lam EW, Thomas NS, Lammers JW, Koenderman L, Coffer PJ. FKHR-L1 can act as a critical effector of cell death induced by cytokine withdrawal: protein kinase B-enhanced cell survival through maintenance of mitochondrial integrity. J Cell Biol 2002;156:531-42

Francois S, El Benna J, Dang PM, Pedruzzi E, GougerotPocidalo MA, Elbim C. Inhibition of neutrophil apoptosis by TLR agonists in whole blood: involvement of the phosphoinositide 3-kinase/Akt and NF-kappaB signaling pathways, leading to increased levels of $\mathrm{Mcl}-1, \mathrm{~A} 1$, and phosphorylated Bad. J Immunol 2005;174:3633-42

Fu Z, Tindall DJ. FOXOs, cancer and regulation of apoptosis Oncogene 2008;27:2312-9

Haegebarth A, Bie W, Yang R, Crawford SE, Vasioukhin V, Fuchs E, Tyner AL. Protein tyrosine kinase 6 negatively regulates growth and promotes enterocyte differentiation in the small intestine. Mol Cell Biol 2006;26:4949-57

Hauck L, Harms C, Grothe D, An J, Gertz K, Kronenberg G, Dietz $R$, Endres $M$, von Harsdorf $R$. Critical role for FoxO3a-dependent regulation of p21CIP1/WAF1 in response to statin signaling in cardiac myocytes. Circ Res 2007;100:50-60

Hong-Geller E, Chaudhary A, Lauer S. Targeting toll-like receptor signaling pathways for design of novel immune therapeutics. Curr Drug Discov Technol 2008;5:29-38 
Kawai T, Sato S, Ishii KJ, Coban C, Hemmi H, Yamamoto M, Terai K, Matsuda M, Inoue J, Uematsu S, Takeuchi O, Akira $\mathrm{S}$. Interferon-alpha induction through Toll-like receptors involves a direct interaction of IRF7 with MyD88 and TRAF6. Nat Immunol 2004;5:1061-8

Krieg AM. Therapeutic potential of Toll-like receptor 9 activation. Nat Rev Drug Discov 2006;5:471-84

Kuo CC, Liang SM, Liang CM. CpG-B oligodeoxynucleotide promotes cell survival via up-regulation of Hsp70 to increase $\mathrm{Bcl}-\mathrm{xL}$ and to decrease apoptosis-inducing factor translocation. J Biol Chem 2006;281:38200-7

Kuo CC, Liang CM, Lai CY, Liang SM. Involvement of heat shock protein (Hsp)90 beta but not Hsp90 alpha in antiapoptotic effect of CpG-B oligodeoxynucleotide. J Immunol 2007;178:6100-8

Lam EW, Francis RE, Petkovic M. FOXO transcription factors: key regulators of cell fate. Biochem Soc Trans 2006;34:722-6

Latz E, Schoenemeyer A, Visintin A, Fitzgerald KA, Monks BG, Knetter CF, Lien E, Nilsen NJ, Espevik T, Golenbock DT. TLR9 signals after translocating from the ER to CpG DNA in the lysosome. Nat Immunol 2004;5:190-8

Lee SS, Kennedy S, Tolonen AC, Ruvkun G. DAF-16 target genes that control $C$. elegans life-span and metabolism. Science 2003;300:644-7

Leonard JP, Link BK, Emmanouilides C, Gregory SA, Weisdorf D, Andrey J, Hainsworth J, Sparano JA, Tsai DE, Horning S, Krieg AM, Weiner GJ. Phase I trial of toll-like receptor 9 agonist PF-3512676 with and following rituximab in patients with recurrent indolent and aggressive non Hodgkin's lymphoma. Clin Cancer Res 2007;13:6168-74

Lim EJ, Lee SH, Lee JG, Kim JR, Yun SS, Baek SH, Lee C. Toll-like receptor 9 dependent activation of MAPK and NF-kB is required for the $\mathrm{CpG}$ ODN-induced matrix metalloproteinase-9 expression. Exp Mol Med 2007;39:239-45

Loeuillet C, Martinon F, Perez C, Munoz M, Thome M, Meylan PR. Mycobacterium tuberculosis subverts innate immunity to evade specific effectors. J Immunol 2006;177:6245-55
Meng Y, Carpentier AF, Chen L, Boisserie G, Simon JM, Mazeron JJ, Delattre JY. Successful combination of local CpG-ODN and radiotherapy in malignant glioma. Int $\mathrm{J}$ Cancer 2005;116:992-7

Skurk C, Maatz H, Kim HS, Yang J, Abid MR, Aird WC, Walsh $\mathrm{K}$. The Akt-regulated forkhead transcription factor FOXO3a controls endothelial cell viability through modulation of the caspase-8 inhibitor FLIP. J Biol Chem 2004;279:1513-25

Srinivasan S, Anitha M, Mwangi S, Heuckeroth RO. Enteric neuroblasts require the phosphatidylinositol 3-kinase/Akt/ Forkhead pathway for GDNF-stimulated survival. Mol Cell Neurosci 2005;29:107-19

Takeshita F, Gursel I, Ishii KJ, Suzuki K, Gursel M, Klinman DM. Signal transduction pathways mediated by the interaction of CpG DNA with Toll-like receptor 9. Semin Immunol 2004;16:17-22

Thome M, Schneider P, Hofmann K, Fickenscher H, Meinl E, Neipel F, Mattmann C, Burns K, Bodmer JL, Schroter M, Scaffidi C, Krammer PH, Peter ME, Tschopp J. Viral FLICE-inhibitory proteins (FLIPs) prevent apoptosis induced by death receptors. Nature 1997;386:517-21

Wang X, Wang Y, Zhang J, Kim HP, Ryter SW, Choi AM. FLIP protects against hypoxia/reoxygenation-induced endotheslial cell apoptosis by inhibiting Bax activation. Mol Cell Biol 2005;25:4742-51

Weigel BJ, Rodeberg DA, Krieg AM, Blazar BR. CpG oligodeoxynucleotides potentiate the antitumor effects of chemotherapy or tumor resection in an orthotopic murine model of rhabdomyosarcoma. Clin Cancer Res 2003;9: 3105-14

Xiao CW, Asselin E, Tsang BK. Nuclear factor kappaBmediated induction of Flice-like inhibitory protein prevents tumor necrosis factor alpha-induced apoptosis in rat granulosa cells. Biol Reprod 2002;67:436-41

Xu Z, Lin S, Wu W, Tan H, Wang Z, Cheng C, Lu L, Zhang $X$. Ghrelin prevents doxorubicin-induced cardiotoxicity through TNF-alpha/NF-kappaB pathways and mitochondrial protective mechanisms. Toxicology 2008;247:133-8 